\title{
Determination of the chemical and hydrothermal stability of the Claus catalyst
}

\author{
Farkhod Yusupov", Murodali Mamanazarov, Bobomurod Xursandov, Gulnoza Baimatova \\ Institute of general and inorganic chemistry of Academy of Science of Republic of Uzbekistan, \\ Tashkent, Uzbekistan
}

\begin{abstract}
The hydrothermal and thermal stability used in the Claus process are important indicators in their workflow. In this work, the stability of two catalyst samples obtained from local raw materials was studied. The samples were processed with $\mathrm{SO} 2 / \mathrm{O} 2$ oxides and water vapor, and their properties were studied. As a result, $\mathrm{SO} 2$ adsorption reduced catalyst activation. The process is reductive; the recovery of catalyst activation by reducing $\mathrm{SO} 2$ in the system has been determined. The stability of sulfation of $\mathrm{MgO} / \mathrm{TiO} 2$ catalyst in comparison with $\mathrm{CaO}$ / V2O5 samples was also determined.
\end{abstract}

\section{Introduction}

The largest sources of air pollution with sulfur compounds are sour gases resulting from the purification of oil and natural gas from hydrogen sulfide and mercaptans and waste gases from non-ferrous metallurgy enterprises. The Claus process is widely used to recover sulfur from such gases. The main disadvantage of modern industrial catalysts for the Claus process based on alumina and titanium dioxide is their deactivation due to the formation of surface sulfates, which leads to a decrease in activity in the Claus reaction and the hydrolysis reaction of COS and $\mathrm{CS}_{2}$.

During operation, industrial catalysts gradually undergo irreversible changes: the specific surface area and total porosity decrease, and the catalytic activity decreases. Of the variety of reasons causing the deactivation of Claus catalysts, the most important are:

- thermal and hydrothermal aging with the transformation of the structure and texture of the catalyst;

- sulfation of the catalyst surface;

- deposition of liquid sulfur on the surface and in the pore volume of the catalyst;

- a decrease in the specific surface area and pore volume of the catalyst due to coke deposits.

The generalization of many years of experience in the industrial operation of alumina catalysts [1-2] shows that

the main responsibility for their deactivation is the change in the chemical properties and texture of $\mathrm{Al}_{2} \mathrm{O}_{3}$ as a result of the formation and deposition of sulfates. Catalyst

\footnotetext{
*Corresponding author: f.yusupov@yandex.ru
} 
samples taken from Claus industrial plants contain sulfates in amounts ranging from $1 \%$ to $10 \%$.

In real conditions [3], the gas mixture, in addition to $\mathrm{H}_{2} \mathrm{~S}$, contains $\mathrm{SO}_{2}$ up to $30 \%$ vol. water vapor. Most oxides of metals of variable valence (for example, $\mathrm{Cu}, \mathrm{Fe}, \mathrm{Co}, \mathrm{Bi}, \mathrm{Mo}$, $\mathrm{Ni}, \mathrm{Sb}$ ), proposed as catalysts for the oxidation of hydrogen sulfide with oxygen and/or sulfur dioxide, under the action of $\mathrm{H}_{2} \mathrm{~S}$ form sulfides and significantly lose their initial activity in the Claus reaction [4] and direct oxidation reactions [5].

With an increase in the ratio of oxidant/hydrogen sulfide, the sulfation of catalysts can occur. It is known from the literature [6] that the sulfur yield in the catalytic stages decreases rapidly with an increase in the content of sulfates in the catalyst. The main reason for the sulfation of catalysts is oxygen and water in the process gases and the difficulty in controlling the $\mathrm{H}_{2} \mathrm{~S} / \mathrm{SO}_{2}$ ratio (excess $\mathrm{SO}_{2}$ ). Sulfation especially significantly reduces the activity of catalysts in low-temperature processes used to carry out the reaction at temperatures below the dew point of sulfur ("Sulfrene"). Under conditions when the $\mathrm{H}_{2} \mathrm{~S} /$ $\mathrm{SO}_{2}$ molar ratio is close to stoichiometric, and there is no oxygen in the gas mixture, an equilibrium concentration of sulfates occurs on the surface of the alumina catalyst in the reaction medium, which, depending on the process temperature, is $2-3 \mathrm{wt} \%$. [7]. The concentration of sulfates on the surface does not practically change during 60 months of operation [8]. In the presence of an excess of an oxidizing agent $\left(\mathrm{SO}_{2}, \mathrm{O}_{2}\right)$ in gas mixtures containing water vapor, the amount of sulfates on the catalyst surface becomes above equilibrium and increases with time. This leads to a gradual loss of catalyst activity, which is restored after regeneration. Thus, the amount of sulfation, and, consequently, the activity of the catalyst, depends on the content of water, sulfur dioxide, and oxygen in the reaction medium.

The presence of water vapor significantly affects the thermodynamics [9] and the kinetics of the process [10]. Water vapor displaces the equilibrium of the reversible Claus reaction; the reaction is inhibited by water vapor due to the competitive adsorption of water on the catalyst's active sites.

In addition, water vapor is the main cause of irreversible catalyst deactivation due to hydrothermal degradation. In most works, when testing catalysts, either deactivation of the catalyst is not taken into account at all [11], or only the sulfation of the catalyst is taken into account by performing an aging test, by treatment with a mixture with different contents of $\mathrm{SO}_{2} / \mathrm{O}_{2}[12,13]$. Only several works propose a technique for simulating hydrothermal aging.

\section{Methods}

The procedure for preparing alumina granule samples is based on the following stages:

- preparation of raw materials and their grinding (disintegration);

- granulation by the pelletizing method;

- physical and chemical processing;

- sieving granules;

- calcination;

- rescreening of granules and packaging of the finished product.

To prepare the catalyst, waste alumina adsorbent is used and in the form of an active component vanadium oxide, calcium hydroxide (1), and magnesium nitrate, titanium dioxide (2). Two different samples were taken: 1 . Titanium dioxide and magnesium oxide as active ingredients; 2 . Vanadium (V) oxide and calcium oxide as active ingredients. The disintegrated product, which mainly consists of particles less than 100 microns in size, while the fraction of the fraction less than 50 microns must be at least $75 \%$, is dosed onto a 
disc granulator at a speed of $\mathrm{n}=18 \mathrm{rpm}$, in which a finely atomized solution is simultaneously fed binder (solution of calcium hydroxide, magnesium nitrate or water).

After the granulator, the resulting intermediate product with a moisture content of 20$25 \%$ enters the reactor of physicochemical treatment, where it is kept in saturated water vapor for at least 2 hours at a temperature of $85-90^{\circ} \mathrm{C}$. Then, the granules are sieved and dried at $120^{\circ} \mathrm{C}$ for $24 \mathrm{~h}$, after which they were calcined at $420-550^{\circ} \mathrm{C}$ in a stream of dried air (dew point is not higher than $-40^{\circ} \mathrm{C}$ ). The pellets were heated at a rate of $70^{\circ} \mathrm{C} / \mathrm{h}$. The duration of calcination after reaching the regime was $4 \mathrm{~h}$.

Hydrolysis of $\mathrm{COS}$ to $\mathrm{CO}_{2}$ and $\mathrm{H}_{2} \mathrm{~S}$ was carried out in a flow reactor. Pure oxides or impregnated sulfated samples were first washed in the reactor for 2 hours at $543 \mathrm{~K}$ : the temperature was set equal to the reaction temperature. Water was transferred from a thermostated saturator in a controlled flow of helium, and $2.7 \mathrm{mmol} \cdot \mathrm{h}-1 \mathrm{COS}$ was added to the feed so that the composition of the feed was as follows: $1 \% \operatorname{COS}, 2.2 \% \mathrm{H}_{2} \mathrm{O}$, and $96.8 \% \mathrm{He}$. It was passed through a temperature-controlled pyrex glass reactor containing a fixed bed of catalyst $(0.025 \mathrm{~g}$ with a particle size in the $0.4 \mu \mathrm{m}$ range). All samples were tested under such conditions to avoid diffusion phenomena. This made it possible to directly compare the conversion (\% conversion per square meter) after 30 minutes of submission. In another series of experiments, $0.2 \% \mathrm{SO}_{2}$ was continuously added to the feed to monitor its toxic effect. After stabilization of activity, $\mathrm{SO}_{2}$ was turned off, and the conversion was measured again.

\section{Results and Discussion}

To determine the resistance of the catalyst to sulfation, the samples were treated with a $\mathrm{SO}_{2} / \mathrm{O}_{2}$ gas mixture at $200-320^{\circ} \mathrm{C}$ for 5 hours.

Figure 1 shows a decrease in the activity of the samples when $\mathrm{SO}_{2}$ is introduced into the feed, regardless of the metal oxide. Since the effect of $\mathrm{SO}_{2}$ is relatively less pronounced on $\mathrm{TiO}_{2}$, the order of activity in the presence of $\mathrm{SO}_{2}$ becomes as follows: $1>2$. Cutting out $\mathrm{SO}_{2}$ in the feed restores the original activity, showing that $\mathrm{SO}_{2}$ poisoning is almost completely reversible (Figure 1). These results well explain why the addition of $\mathrm{SO}_{2}$ to the feed gas partially inhibits COS hydrolysis. $\mathrm{SO}_{2}$ and $\mathrm{COS}$ are competitively adsorbed on the same main Bronsted centers, and the former are adsorbed more strongly than the latter.

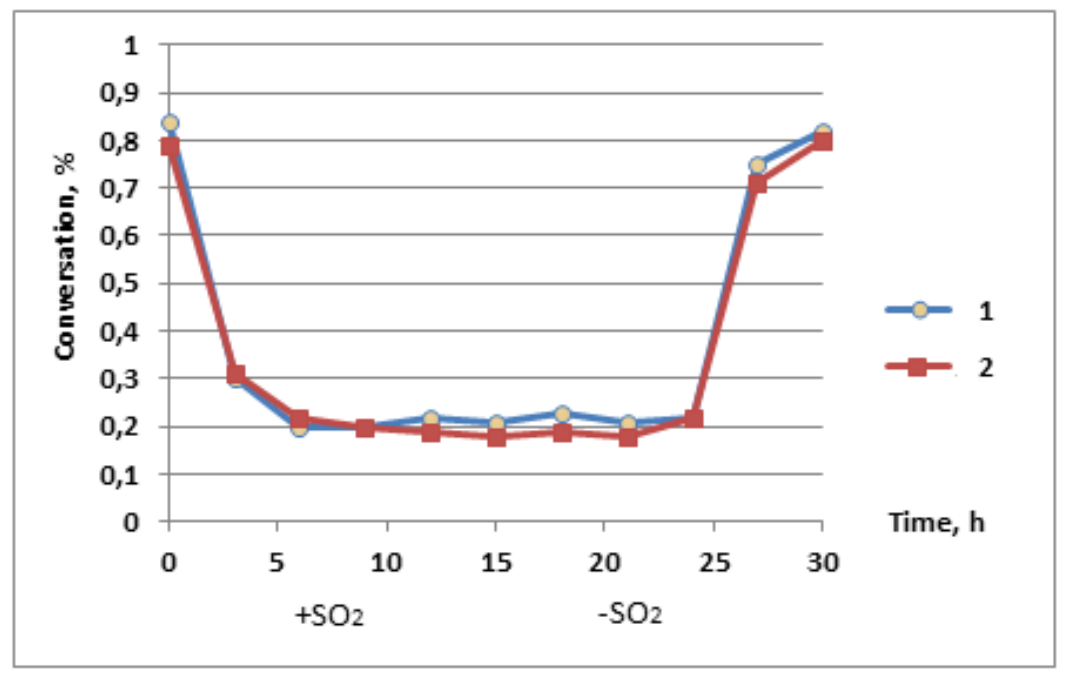

Fig.1. Effect of $\mathrm{SO}_{2}(0.2 \%)$ on the hydrolysis of $\mathrm{COS}$ at $503 \mathrm{~K}\left(1 . \mathrm{MgO}+\mathrm{TiO}_{2} ; 2 . \mathrm{CaO}+\mathrm{V}_{2} \mathrm{O}_{5}\right)$. 


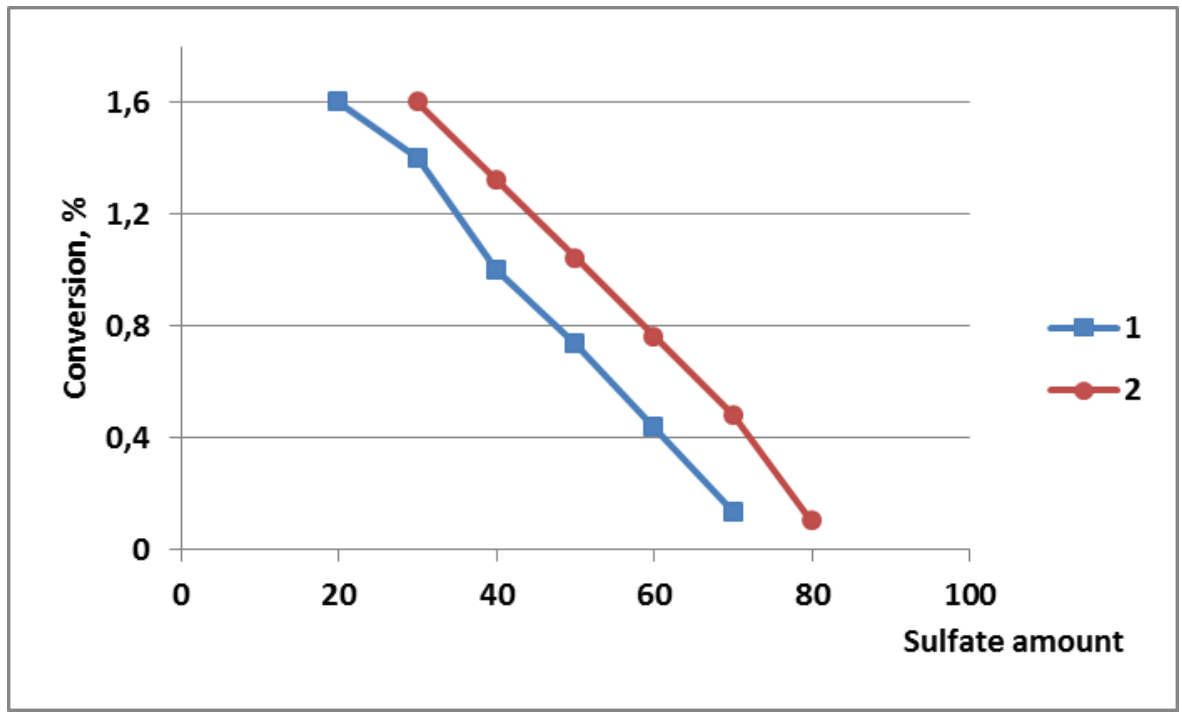

Fig.2. Dependence of conversion on sulfation $\left(1 . \mathrm{MgO}+\mathrm{TiO}_{2} ; 2 . \mathrm{CaO}+\mathrm{V}_{2} \mathrm{O}_{5}\right)$.

The loading of pure oxides with sulfates decreases the catalytic activity regardless of the sample (Fig. 2). When the amount of sulfate reaches $0.8 \mathrm{mmol} \cdot \mathrm{m}-2$, the degree of deactivation changes in the following order: $2>1$ so that $\mathrm{V}_{2} \mathrm{O}_{5}$ becomes more active than $\mathrm{TiO}_{2}$. Sulfate particles are well characterized by an IR band of $1360 \mathrm{~cm}-1$ on samples activated for 2 hours at $723 \mathrm{~K}$, and their number is proportional to its intensity [14]. We measured the sulfate concentration by this spectroscopic method before and after the reaction. The amounts of sulfate did not appear to change, indicating that they are stable and not reduced by $\mathrm{H}_{2} \mathrm{~S}$, one of the reaction products under the reaction conditions used.

The pulse introduction of oxygen into the gas supply during the $\mathrm{OH}$ reaction on pure oxides leads to the same effect as presulfation: an irreversible decrease in catalytic activity is observed. For two samples, it was completed after two pulses and is much higher on $\mathrm{TiO}_{2}$ $(68 \%)$ than on $\mathrm{V}_{2} \mathrm{O}_{5}(55 \%)$. In the IR spectra of the samples after these experiments, there are characteristic bands of the already described sulfate forms. Their number changes in the following order: $\mathrm{TiO}_{2}>\mathrm{V}_{2} \mathrm{O}_{5}$ and corresponds well to the observed deactivation. It is important to note that pulsed sulfation is more significant on $\mathrm{TiO}_{2}$ than the less alkaline sample. Under real conditions, alumina undergoes various types of aging (sulfation, reduced surface area), either due to normal use or for some random reason. To better understand the performance of a catalyst, it is necessary to look at the aging process.

Therefore, we have developed the following procedure to simulate aging: treatment at $400-550^{\circ} \mathrm{C}$ for 12 hours in a stream of nitrogen with $50 \%$ steam.

Table 1. Results of hydrothermal aging of the catalyst (depending on time).

\begin{tabular}{|l|c|c|c|c|c|}
\hline Time, $\mathrm{min}$ & 0 & 6 & 8 & 10 & 12 \\
\hline Surface area, $\mathrm{m}^{2} / \mathrm{g}$ & 290 & 250 & 200 & 165 & 130 \\
\hline Mechanical strength $\mathrm{MPa}$ & 8 & 6.5 & 5 & 4 & 3.5 \\
\hline Total pore volume, $\mathrm{cm}^{3} / \mathrm{g}$ & 0.55 & 0.48 & 0.42 & 0.38 & 0.33 \\
\hline Density, $\mathrm{g} / \mathrm{cm}^{3}$ & 0.69 & 0.70 & 0.72 & 0.73 & 0.75 \\
\hline Macropores, $\mathrm{g} / \mathrm{cm}^{3}$ & 0.31 & 0.32 & 0.30 & 0.29 & 0.29 \\
\hline Micropores, $\mathrm{g} / \mathrm{cm}^{3}$ & 0.24 & 0.16 & 0.12 & 0.11 & 0.4 \\
\hline
\end{tabular}

The main consequence of this is that the catalyst surface area is reduced to $130 \mathrm{~m}^{2} / \mathrm{g}$, which is close to the level at which users recharge the alumina in their units. This decrease is 
caused by the collapse of micropores with a diameter of less than $40 \mathrm{~A}$, as seen from the porosity distribution measured by mercury porosimetry. No macropore modification is observed.

Table 2. Results of hydrothermal aging of the catalyst (depending on temperature).

\begin{tabular}{|l|c|c|c|c|c|}
\hline Temperature, ${ }^{\circ} \mathrm{C}$ & 200 & 230 & 260 & 290 & 320 \\
\hline Surface area, $\mathrm{m}^{2} / \mathrm{g}$ & 290 & 250 & 200 & 165 & 130 \\
\hline Mechanical strength MPa & 8 & 6.5 & 5 & 4 & 3.5 \\
\hline Total pore volume, $\mathrm{cm}^{3} / \mathrm{g}$ & 0.55 & 0.48 & 0.42 & 0.38 & 0.33 \\
\hline Density, $\mathrm{g} / \mathrm{cm}^{3}$ & 0.69 & 0.70 & 0.72 & 0.73 & 0.75 \\
\hline Macropores, $\mathrm{g} / \mathrm{cm}^{3}$ & 0.31 & 0.32 & 0.30 & 0.29 & 0.29 \\
\hline Micropores, $\mathrm{g} / \mathrm{cm}^{3}$ & 0.24 & 0.16 & 0.12 & 0.11 & 0.4 \\
\hline
\end{tabular}

\section{Conclusions}

This study showed that the catalytic activities of the alumina and titanium-based system were decreased due to hydrothermal aging and sulfur poisoning. $\mathrm{SO}_{2}$ adsorption occurs in the same areas as during COS hydrolysis. These results explain why the addition of $\mathrm{SO}_{2}$ to the test gas partially inhibits conversion. The reversibility of $\mathrm{SO}_{2}$ poisoning occurs due to the desorption of hydrosulfite species when $\mathrm{SO}_{2}$ is turned off and also due to the reaction between residual hydrogen sulfite species and $\mathrm{H}_{2} \mathrm{~S}$ (Claus reaction) formed during the hydrolysis of COS.

The sulfation of metal oxides poisons the main centers and creates Bronsted acidity, which explains the deactivation of the catalyst (23). The sulfate compounds are quite stable even in the presence of water under the conditions used. It was found that samples based on titanium and magnesium oxide are more resistant to sulfation.

\section{References}

1. R. Kettner and T. Lubcke. Sulphur, Hu H, Yang Q, Lu X, Wang WC, Wang SS, Fan MH Crit Rev Environ Sci Tech 40. p 452. (1982)

2. Wang XH, Wang AQ, Li N, Wang XD, Liu Z, Zhang T Ind Eng Chem Prod Res Dev 45. p 4582. (2006)

3. Z.M. George, J. Catal, 35. p. 218. (1974)

4. J. West, B.P. Williams, N.C. Young, C. Rhodes and G.J. Hutchings, Catal. Lett. 74. p 111. (2001).

5. C. Rhodes, S.A. Riddel, J. West, B.P. Williams and G.J. Hutchings, Catal. Today 59. p 443. (2000).

6. M. M. Haque, A. Khan, K. Umar, N. A. Mir, M. Muneer, T. Harada, and M. Matsumura, Energy Environ. Focus 2, p 73 (2013)

7. J.C. Lavalley, K. Aboulayt, M. Lion, J. Bachelier, J.L. Hebrard and F.Luck. The Division of Petroleum Chemistry Inc. ACS Preprints of the Symposium on NO, and SO2 Control in Stationary Sources, $36 \mathrm{p}$ 43. (1991).

8. H. Z. Zhang and J. F. Banfield, J. Mater. Res. 15, p 437 (2000).

9. Laprdrix, E. Justin, I. Costentin, G. Saur, O. Lavalley, J. C. Aboulayt, A. Ray, J. L. Nedez, C. Comparative study of CS2 hydrolysis catalyzed by alumina and titania. Appl. Catal, B 17, pp 167-173. (1998).

10. Oleshko V.P, Lunina Ye.V, Golubev V.B, Bychkova T.V.Nekrasov L.I. Zhurn. fiz. khimii.T. 55. (5). p 1160. (1981). 
11. Ivanova A.S., Al'kaeva E.M., Mastikhin V.M., Pauksh·tis E.A., Kryukova G.N. Kinetika i kataliz.37.(3). P. 450. (1996).

12. Bashkova S, Baker F S, Wu X X, Armstrong T R, Schwartz V. Carbon, 45 (6), p 1354. (2007). 